\title{
Optimal partial regularity for nonlinear sub-elliptic systems with Dini continuous coefficients in Carnot groups
}

Jialin Wang ${ }^{*}$ and Dongni Liao

\section{"Correspondence:}

jialinwang1025@hotmail.com School of Mathematics and Computer Science, Gannan Normal University, Ganzhou, Jiangxi 341000, P.R. China

\begin{abstract}
This paper is concerned with the interior regularity for nonlinear sub-elliptic systems with Dini continuous coefficients under superquadratic controllable growth conditions in Carnot groups. We adapt the technique of the $\mathcal{A}$-harmonic approximation to the case of sub-elliptic systems in divergence form, and we show a partial regularity result for weak solutions. In particular, our result is optimal in the sense that in the case of Hölder continuous coefficients we obtain directly the optimal Hölder exponent for the horizontal gradient of weak solutions on its regular set.

Keywords: optimal partial regularity; Dini continuity coefficient; controllable growth condition; Carnot group; sub-elliptic system
\end{abstract}

\section{Introduction and statements of main results}

In this paper, we consider the following nonlinear sub-elliptic systems under superquadratic controllable growth $(m>2)$ conditions in Carnot groups $G$ in divergence form:

$$
-\sum_{i=1}^{k} X_{i} A_{i}^{\alpha}(\xi, u, X u)=B^{\alpha}(\xi, u, X u) \quad \text { in } \Omega, \alpha=1,2, \ldots, N,
$$

where $\Omega$ is a bounded domain in $G, X=\left\{X_{1}, \ldots, X_{k}\right\}$ with $X_{i}(i=1, \ldots, k)$ to see the next section (2.1) below, $u=\left(u^{1}, \ldots, u^{N}\right): \Omega \rightarrow R^{N}, A_{i}^{\alpha}(\xi, u, p): \Omega \times R^{N} \times R^{k \times N} \rightarrow R^{k \times N}$, and $B^{\alpha}(\xi, u, p): \Omega \times R^{N} \times R^{k \times N} \rightarrow R^{N}$.

Under the coefficients $A_{i}^{\alpha}$ assumed to be Dini continuous, the purpose of this paper is to establish optimal partial regularity to the system (1.1) under the superquadratic controllable growth conditions. Such an assumption is much weaker than the assumption of Hölder continuity; see $[1,2]$ for the case of sub-elliptic systems. More precisely, we assume for the continuity of $A_{i}^{\alpha}$ with respect to the variables $(\xi, u)$ that

$$
(1+|p|)^{-\frac{m}{2}}\left|A_{i}^{\alpha}(\xi, u, p)-A_{i}^{\alpha}(\tilde{\xi}, \tilde{u}, p)\right| \leq \kappa(|u|) \mu(d(\xi, \tilde{\xi})+|u-\tilde{u}|)
$$

for all $\xi, \tilde{\xi} \in \Omega, u, \tilde{u} \in R^{N}$, and $p \in R^{k \times N}$, where $\kappa:(0,+\infty) \rightarrow[1,+\infty)$ is nondecreasing, and $\mu:(0,+\infty) \rightarrow[0,+\infty)$ is nondecreasing and concave with $\mu(0+)=0$. We also require

\section{Springer}


that $r \rightarrow r^{-\gamma} \mu(r)$ is nonincreasing for some $\gamma \in(0,1)$ and that

$$
M(r)=\int_{0}^{r} \frac{\mu(\rho)}{\rho} d \rho<\infty, \quad \text { for some } r>0 .
$$

We employ the method of an $\mathcal{A}$-harmonic approximation to investigate the sub-elliptic system (1.1) under this weaker assumption, and we establish optimal partial regularity results: Roughly speaking, assume additionally to the standard hypotheses (see precisely (H1), (H2), and (H4) below) that $(1+|p|)^{-\frac{m}{2}} A_{i}^{\alpha}(\xi, u, p)$ satisfies (1.2) and (1.3). Let $u \in H W^{1, m}\left(\Omega, R^{N}\right)$ be a weak solution of (1.1). Then $u$ is of class $C^{1}$ outside a closed singular set Sing $u \subset \Omega$ of Haar measure 0. Furthermore, for $\xi_{0} \in \Omega \backslash \operatorname{Sing} u$, the derivative $X u$ of $u$ has the modulus of continuity $r \rightarrow M(r)$ in a neighborhood of $\xi_{0}$. Our result is optimal in the sense that when $\mu(\rho)=\rho^{\gamma}, 0<\gamma<1$, we have $M(r)=\gamma^{-1} r^{\gamma}$, and $\Gamma^{1, \gamma}$ regularity is known to be optimal in that case; see [1].

As is well known, one, in general, cannot expect that weak solutions of the sub-elliptic system (1.1) will be classical, i.e. $C^{2}$-solutions, even under reasonable assumptions on $A_{i}^{\alpha}$ and $B^{\alpha}$. This was first shown by De Giorgi [3] in the Euclidean space; we also refer the reader to Giaquinta [4], and $\mathrm{Chen}$ and $\mathrm{Wu}$ [5] for further discussion and additional examples. Then the goal is to establish partial regularity theory. Moreover, a new method called $\mathcal{A}$-harmonic approximation technique is introduced by Duzaar and Steffen in [6], and simplified by Duzaar and Grotowski in [7], to study elliptic systems with quadratic growth case. Then similar results have been proved for more general $A_{i}^{\alpha}$ or $B^{\alpha}$ in the Euclidean setting; see [8-11] for Hölder continuous coefficients, and [12-14] for Dini continuous coefficients.

However, turning to sub-elliptic equations and systems in Carnot groups, some new difficulties will arise due to non-commutativity of the horizontal vectors $X_{i}$. With regard to cases of sub-elliptic equations, we refer the reader to Domokos [15-17], Capogna [18, 19], Manfredi and Mingione [20], and Mingione et al. [21] for more interesting results and details. Several results were focused on sub-elliptic systems. Capogna and Garofalo [22], and Shores [23] considered the quadratic growth case, and Föglein [24], Wang and Niu [1], and Wang and Liao [2] treated non-quadratic cases. We note that the assumption of Hölder continuity on the coefficients $A_{i}^{\alpha}$ is required for those regularity results of subelliptic systems mentioned above.

When the assumption of Hölder continuity of $A_{i}^{\alpha}$ is weakened to Dini continuity, one may ask how to establish partial regularity for the nonlinear sub-elliptic systems under superquadratic controllable growth conditions in the Carnot groups. This paper is devoted to this topic. To define weak solutions to (1.1), we assume the following structure conditions on $A_{i}^{\alpha}$ and $B^{\alpha}$ :

(H1) The term $A_{i}^{\alpha}(\xi, u, p)$ is differentiable in $p$, and there exists some constant $L$ such that

$$
\left|A_{i, p_{\beta}^{j}}^{\alpha}(\xi, u, p)\right| \leq L\left(1+|p|^{2}\right)^{\frac{m-2}{2}}, \quad(\xi, u, p) \in \Omega \times R^{N} \times R^{k \times N}, m>2,
$$

where we write $A_{i, p_{\beta}^{\prime}}^{\alpha}(\xi, u, p)=\frac{\partial A_{i}^{\alpha}(\xi, u, p)}{\partial p_{\beta}^{j}}$.

(H2) The term $A_{i}^{\alpha}(\xi, u, p)$ is uniformly elliptic, i.e. for some $\lambda>0$ we have

$$
A_{i, p_{\beta}^{j}}^{\alpha}(\xi, u, p) \eta_{i}^{\alpha} \eta_{j}^{\beta} \geq \lambda(1+|p|)^{m-2}|\eta|^{2}, \quad \forall \eta \in R^{k \times N} .
$$


(H3) There exist a modulus of continuity $\mu:(0,+\infty) \rightarrow[0,+\infty)$ and a nondecreasing function $\kappa:[0,+\infty) \rightarrow[1,+\infty)$ such that

$$
(1+|p|)^{-\frac{m}{2}}\left|A_{i}^{\alpha}(\xi, u, p)-A_{i}^{\alpha}(\tilde{\xi}, \tilde{u}, p)\right| \leq \kappa(|u|) \mu(d(\xi, \tilde{\xi})+|u-\tilde{u}|) .
$$

(H4) The term $B^{\alpha}$ satisfies the superquadratic controllable growth condition

$$
\left|B^{\alpha}(\xi, u, p)\right| \leq c\left(1+|u|^{r-1}+|p|^{m(r-1) / r}\right),
$$

where $c$ is a positive constant, and $r=\frac{m Q}{Q-m}$ if $m<Q$; or $Q \leq r<+\infty$ if $m=Q$.

Without loss of generality we can assume $\kappa \geq 1$ and that

( $\mu 1) \quad \mu$ is nondecreasing with $\mu(0+)=0, \mu(1)=1$;

( $\mu 2) \mu$ is concave, in the proof of the regularity result we have to require that $r \rightarrow r^{-\gamma} \mu(r)$ is nonincreasing for some exponent $\gamma \in(0,1)$;

( $\mu 3)$ Dini's condition $M(r)=\int_{0}^{r} \frac{\mu(\rho)}{\rho} d \rho<\infty$ for some $r>0$.

Note that $(\mathrm{H} 1)$ one infers

$$
\left|A_{i}^{\alpha}(\xi, u, p)-A_{i}^{\alpha}(\xi, u, \tilde{p})\right| \leq C(L)\left(1+|p|^{2}+|\tilde{p}|^{2}\right)^{\frac{m-2}{2}}|p-\tilde{p}|,
$$

and there exists a continuously nonnegative and bounded function $\omega(s, t):[0, \infty) \times$ $[0, \infty) \rightarrow[0, \infty)$, where $\omega(s, 0)=0$ for all $s$, and $\omega(s, t)$ is monotonously nondecreasing in $s$ for fixed $t ; \omega(s, t)$ is concave and monotonously nondecreasing in $t$ for fixed $s$, such that for all $(\xi, u, p),(\tilde{\xi}, \tilde{u}, \tilde{p}) \in \Omega \times R^{N} \times R^{k \times N}$,

$$
\left|A_{i, p_{\beta}^{j}}^{\alpha}(\xi, u, p)-A_{i, p_{\beta}^{j}}^{\alpha}(\xi, u, \tilde{p})\right| \leq C\left(1+|p|^{2}+|\tilde{p}|^{2}\right)^{\frac{m-2}{2}} \omega\left(|p|,|p-\tilde{p}|^{2}\right) .
$$

Further (H2) allows us to deduce the following inequality; see [1] for more details:

$$
\left(A_{i}^{\alpha}(\xi, u, p)-A_{i}^{\alpha}(\xi, u, \tilde{p})\right)(p-\tilde{p}) \geq \lambda_{0}\left(|p-\tilde{p}|^{2}+|p-\tilde{p}|^{m}\right)
$$

with a positive constant $\lambda_{0}$.

To obtain partial regularity for weak solutions, the key point is to establish an excessdecay estimate for the functional

$$
\Phi\left(\xi_{0}, \rho, p_{0}\right)=f_{B_{\rho}\left(\xi_{0}\right)}\left(\left|X u-p_{0}\right|^{2}+\left|X u-p_{0}\right|^{m}\right) d \xi,
$$

here we write $f_{B_{\rho}\left(\xi_{0}\right)} u d \xi=\left|B_{r}\left(\xi_{0}\right)\right|_{G}^{-1} \int_{B_{\rho}\left(\xi_{0}\right)} u d \xi$, which itself leads to the continuity of the horizontal gradient $X u$ of the weak solution $u$ via the integral characterization of continuity by Campanato; see [19]. Since the non-commutative basic vector fields $\left\{X_{i}\right\}$ of Lie algebras corresponding to the Carnot group are more complicated than gradient vector fields $\left\{\frac{\partial}{\partial x_{i}}\right\}$ in the Euclidean space, we have to find different auxiliary functions in treating subelliptic systems. Besides, the non-horizontal derivatives of weak solutions will appear in the Taylor type formula on the Carnot groups and cannot be controlled under the present hypotheses. So the method employing Taylor's formula in [7] cannot trivially be adapted to our cases. We apply the Poincaré type inequality in [25] instead of Taylor's formula, and we establish the main results. 
Theorem 1 Assume that coefficients $A_{i}^{\alpha}$ and $B^{\alpha}$ satisfy (H1)-(H4), $(\mu 1)-(\mu 3)$. Let $u \in$ $H W^{1, m}\left(\Omega, R^{N}\right)$ be a weak solution to the system (1.1), i.e.,

$$
\int_{\Omega} A_{i}^{\alpha}(\xi, u, X u) X_{i} \varphi^{\alpha} d \xi=\int_{\Omega} B^{\alpha}(\xi, u, X u) \varphi^{\alpha} d \xi \quad \forall \varphi \in C_{0}^{\infty}\left(\Omega, R^{N}\right)
$$

Then there exists a relatively closed set $\operatorname{Sing} u \subset \Omega$ such that $u \in C^{1}\left(\Omega \backslash \operatorname{Sing} u, R^{N}\right)$. Furthermore, Sing $u \subset \Sigma_{1} \cup \Sigma_{2}$ and Haar meas $(\Omega \backslash \operatorname{Sing} u)=0$, where

$$
\begin{aligned}
& \Sigma_{1}=\left\{\xi_{0} \in \Omega: \sup _{r>0}\left(\left|u_{\xi_{0}, r}\right|+\left|(X u)_{\xi_{0}, r}\right|\right)=\infty\right\}, \\
& \Sigma_{2}=\left\{\xi_{0} \in \Omega: \lim _{r \rightarrow 0^{+}} \inf f_{B_{r}\left(\xi_{0}\right)}\left|X u-(X u)_{\xi_{0}, r}\right|^{2} d \xi>0\right\} .
\end{aligned}
$$

In addition, for $\tau \in[\gamma, 1)$ and $\xi_{0} \in \Omega \backslash$ Sing $u$ the horizontal derivative Xu has the modulus of continuity $r \rightarrow r^{\tau}+M(r)$ in a neighborhood of $\xi_{0}$.

\section{Preliminaries}

A Carnot group $G$ of step $r$ is a simply connected, nilpotent Lie group whose Lie algebra $\mathcal{G}$ admits a stratification, i.e., $\mathcal{G}=V^{1} \oplus V^{2} \oplus \cdots \oplus V^{r}$, such that $\left[V^{1}, V^{j}\right]=V^{j+1}, j=1, \ldots, r-1$, and $\left[V^{1}, V^{r}\right]=\{0\}$. Let $X_{i}^{l}$ denote a left-invariant basis vector field of $V^{l}$ with $1 \leq l \leq r$ and $1 \leq i \leq m_{l}$, where $m_{l}$ is the dimension of $V^{l}$. For the sake of simplicity, we let $X_{i}=X_{i}^{1}$, $k=m_{1}, X=\left\{X_{1}, \ldots, X_{k}\right\}$ be the basis of left-invariant vector fields of $V^{1}$, and denote by $\nabla_{G}=$ $\left(X_{1}, \ldots, X_{k}\right)$ the sub-elliptic gradient. We will say that $X_{i}(i=1, \ldots, k)$ are the horizontal vector fields with the form

$$
X_{i}=\partial_{i}+\sum_{j=i+1}^{n} a_{i j}(\xi) \partial_{j}, \quad X_{i}(0)=\partial_{i}
$$

where $a_{i j}(\xi)$ is a polynomial in $\xi$. For a vector valued function $u=\left(u^{1}, \ldots, u^{N}\right): G \mapsto R^{N}$, we let $X_{i} u^{\alpha}(i=1, \ldots, k, \alpha=1, \ldots, N)$ be a horizontal direction derivative, and say that $X u$ is the horizontal Jacobian with entries $X_{i} u^{\alpha}$.

Denoting

$$
\xi=\left(\xi^{1}, \xi^{2}, \ldots, \xi^{r}\right)=\left(x_{1}^{1}, x_{2}^{1}, \ldots, x_{m_{1}}^{1} ; x_{1}^{2}, \ldots, x_{m_{2}}^{2} ; \ldots ; x_{1}^{r}, \ldots, x_{m_{l}}^{r}\right) \in G,
$$

the distance from the origin is defined by

$$
d(\xi)=\left[\sum_{l=1}^{r}\left(\sum_{i=1}^{m_{l}}\left|x_{i}^{l}\right|^{2}\right)^{\frac{r !}{l}}\right]^{\frac{1}{2 r !}} .
$$

For any $\xi, \eta \in G$, we set $d(\xi, \eta)=d\left(\eta^{-1} \circ \xi\right)$, where $\eta^{-1}=-\eta=\left(-\eta^{1}, \ldots,-\eta^{r}\right)$ is the inverse of $\eta$, and $\circ$ is the multiplication rule in $G$ defined by $\xi \circ \widetilde{\xi}=\xi+\widetilde{\xi}+P(\xi, \widetilde{\xi}), \xi, \widetilde{\xi} \in G$, where $P: G \times G \mapsto G$ has polynomial components.

We denote by $\omega_{G}=\left|B\left(\xi_{0}, 1\right)\right|_{G}$ the volume of unit pseudo-ball. Then the volume of the pseudo-ball $B_{R}\left(\xi_{0}\right)=\left\{\eta \in G \mid d\left(\xi_{0}, \xi\right)<R\right\}$ is given by $\left|B\left(\xi_{0}, R\right)\right|_{G}=\omega_{G} R^{Q}$, where $Q=$ $\sum_{l=1}^{r} l m_{l}$ is the homogeneous dimension of $G$. 
The horizontal Sobolev space $H W^{1, p}(\Omega)(1 \leq p<\infty)$ is defined as

$$
H W^{1, p}(\Omega)=\left\{u \in L^{p}(\Omega): X_{i} u \in L^{p}(\Omega), i=1,2, \ldots, k\right\}
$$

Then $H W^{1, p}(\Omega)$ is a Banach space with the norm

$$
\|u\|_{H W^{1, p}(\Omega)}=\|u\|_{L^{p}(\Omega)}+\sum_{i=1}^{k}\left\|X_{i} u\right\|_{L^{p}(\Omega)}
$$

Lu [25] showed the following Poincaré type inequality related to Hörmander's vector fields for $u \in H W^{1, q}\left(B_{R}\left(\xi_{0}\right)\right), 1<q<Q, 1 \leq p \leq \frac{q Q}{Q-q}$ :

$$
\left(f_{B_{R}\left(\xi_{0}\right)}\left|u-u_{\xi_{0}, R}\right|^{p} d \xi\right)^{\frac{1}{p}} \leq C_{p} R\left(f_{B_{R}\left(\xi_{0}\right)}|X u|^{q} d \xi\right)^{\frac{1}{q}}
$$

where $u_{\xi_{0}, R}=f_{B_{r}\left(\xi_{0}\right)} u d \xi=\left|B_{r}\left(\xi_{0}\right)\right|_{G}^{-1} \int_{B_{r}\left(\xi_{0}\right)} u d \xi$. Note the fact that the horizontal vectors $X_{i}$ defined in (2.1) fit the Hörmander's vector fields, and that (2.4) is valid for $p=q=m$ $(\geq 2)$.

Let $0<m, q<\infty, f \in L^{m}(\Omega) \cap L^{q}(\Omega)$. By Hölder's inequality, it follows for any $r$ with $m<r<q$

$$
\|f\|_{r} \leq\|f\|_{m}^{1-\alpha}\|f\|_{q}^{\alpha}
$$

where $0<\alpha<1$ with $\frac{1}{r}=\frac{1-\alpha}{m}+\frac{\alpha}{q}$.

Following [12], for technical convenience, letting $\eta(t)=\mu^{2}(\sqrt{t})$, we have the corresponding properties for $\eta$ : $(\eta 1) \eta$ is continuous, nondecreasing and $\eta(0)=0 ;(\eta 2) \eta$ is concave, and $r \rightarrow r^{-\gamma} \eta(r)$ is nonincreasing for some exponent $\gamma \in(0,1) ;(\eta 3) H(r):=4 M^{2}(\sqrt{r})=$ $\left[\int_{0}^{r} \frac{\sqrt{\eta(\rho)}}{\rho} d \rho\right]^{2}<\infty$ for some $r>0$. Changing $\kappa$ by a constant, but keeping $\kappa \geq 1$, we can assume that $(\eta 4) \eta(1)=1$, implying $\eta(t) \geq t$ for $t \in[0,1]$.

From the fact that $\eta$ is nondecreasing we conclude $s \eta(t) \leq s \eta(t)$ for all $0 \leq t \leq s$. We use the nonincreasing property of $r \mapsto \frac{\eta(r)}{r}$ and $\eta(1) \leq 1$. Combining both cases we get

$$
s \eta(t) \leq s \eta(s)+t, \quad s \in[0,1], t>0 .
$$

From $(\eta 2)$, we deduce for $\theta \in(0,1), t>0, j \in N \cup\{0\}$,

$$
\frac{2}{\gamma}\left(1-\theta^{\gamma}\right) \eta^{1 / 2}\left(\theta^{2 j} t\right)=\int_{\theta^{2(j+1)} t}^{\theta^{2 j} t} \tau^{\frac{\gamma}{2}-1} \frac{\eta^{1 / 2}\left(\theta^{2 j} t\right)}{\left(\theta^{2 j} t\right)^{\gamma / 2}} d \tau \leq \int_{\theta^{2(j+1)} t}^{\theta^{2 j} t} \frac{\eta^{1 / 2}(\tau)}{\tau} d \tau
$$

which implies

$$
\sum_{j=0}^{\infty} \eta^{1 / 2}\left(\theta^{2 j} t\right) \leq \frac{\gamma}{2\left(1-\theta^{\gamma}\right)} \int_{0}^{t} \frac{\eta^{1 / 2}(\tau)}{\tau} d \tau=\frac{\gamma}{2\left(1-\theta^{\gamma}\right)} H^{1 / 2}(t)
$$

It yields particularly that $\eta(t) \leq \frac{\gamma^{2}}{4} H(t)$ for all $t \leq 0$, and $t \mapsto t^{-\gamma} H(t)$ is also nonincreasing. 
In the sequel, we let $\rho_{1}(s, t)=(1+s+t)^{-1} \kappa(s+t)^{-1}$, and $K_{1}(s, t)=(1+t)^{2 m} \kappa^{4}(s+t)$ for $s, t \geq 0$. The constant $C$ may vary from line to line. Note that $\rho_{1} \leq 1$ and that $s \rightarrow \rho_{1}(s, t)$, $t \rightarrow \rho_{1}(s, t)$ are nonincreasing functions.

\section{Caccioppoli type inequality}

In this section, we begin by introducing the $\mathcal{A}$-harmonic approximation lemma, and the proof is similar to [7], also see Föglein [24] with $p=2$ in the Heisenberg group. We mainly prove the Caccioppoli type inequality for weak solutions of the systems (1.1) with controllable growth conditions.

Lemma 2 Let $\lambda$ and $L$ be fixed positive numbers, and $k, N \in \mathbb{N}$ with $k \geq 2$. Assume for any given $\varepsilon>0$, there exists $\delta=\delta(k, N, \lambda, \varepsilon) \in(0,1]$ with the following properties:

(I) for any $\mathcal{A} \in \operatorname{Bil}\left(\mathbb{R}^{k \times N}\right)$ satisfying

$$
\mathcal{A}(v, v) \geq \lambda|v|^{2} \quad \text { and } \quad \mathcal{A}(v, \bar{v}) \leq L|v \| \bar{v}|, \quad v, \bar{v} \in \mathbb{R}^{k \times N},
$$

(II) for any $w \in H W^{1,2}\left(B_{\rho}\left(\xi_{0}\right), \mathbb{R}^{N}\right)$ satisfying

$$
\begin{aligned}
& f_{B_{\rho}\left(\xi_{0}\right)}|X w|^{2} d \xi \leq 1 \text { and } \\
& \left|f_{B_{\rho}\left(\xi_{0}\right)} \mathcal{A}(X w, X \varphi) d \xi\right| \leq \delta \sup _{B_{\rho}\left(\xi_{0}\right)}|X \varphi|, \quad \forall \varphi \in C_{0}^{1}\left(B_{\rho}\left(\xi_{0}\right), \mathbb{R}^{N}\right) .
\end{aligned}
$$

Then there exists an $\mathcal{A}$-harmonic function h such that

$$
f_{B_{\rho}\left(\xi_{0}\right)}|X h|^{2} d \xi \leq 1 \quad \text { and } \quad \rho^{-2} f_{B_{\rho}\left(\xi_{0}\right)}|h-w|^{2} d \xi \leq \varepsilon
$$

Shores in [23] established the following prior estimate for the weak solution $u$ to the sub-elliptic systems with constant coefficients in Carnot groups:

$$
\sup _{B_{\rho / 2}\left(\xi_{0}\right)}\left(|u|^{2}+\rho^{2}|X u|^{2}+\rho^{4}\left|X^{2} u\right|^{2}\right) \leq C_{0} \rho^{2} f_{B_{\rho}\left(\xi_{0}\right)}|X u|^{2} d \xi .
$$

Lemma 3 Let $u \in H W^{1, m}\left(\Omega, \mathbb{R}^{N}\right)$ be a weak solution to the system (1.1) under the conditions (H1)-(H4) and ( $\mu 1)-(\mu 3)$. Then for every $\xi_{0}=\left(\xi_{0}^{1}, \xi_{0}^{2}, \ldots, \xi_{0}^{r}\right) \in \Omega, u_{0} \in \mathbb{R}^{N}, p_{0} \in \mathbb{R}^{k \times N}$ and $0<\rho<R<\rho_{1}^{m / 2}\left(\left|u_{0}\right|,\left|p_{0}\right|\right) \leq 1$ such that $B_{R}\left(\xi_{0}\right) \subset \subset \Omega$, the inequality

$$
\begin{aligned}
& \int_{B_{\rho}\left(\xi_{0}\right)}\left(\left|X u-p_{0}\right|^{2}+\left|X u-p_{0}\right|^{m}\right) d \xi \\
& \leq \frac{C_{c}}{(R-\rho)^{2}} \int_{B_{R}\left(\xi_{0}\right)}\left|u-u_{0}-\left(\xi^{1}-\xi_{0}^{1}\right) p_{0}\right|^{2} d \xi \\
& \quad+\frac{C_{c}}{(R-\rho)^{m}} \int_{B_{R}\left(\xi_{0}\right)}\left|u-u_{0}-\left(\xi^{1}-\xi_{0}^{1}\right) p_{0}\right|^{m} d \xi \\
& \quad+C_{c} \omega_{G} R^{Q} K_{1}\left(\left|u_{0}\right|,\left|p_{0}\right|\right) \eta\left(R^{2}\right)+C_{c}\left[\int_{B_{R}\left(\xi_{0}\right)}\left(1+|u|^{r}+|X u|^{m}\right) d \xi\right]^{\frac{m(r-1)}{r(m-1)}}
\end{aligned}
$$

holds, where $\xi^{1}=\left(\xi_{1}^{1}, \xi_{2}^{1}, \ldots, \xi_{k}^{1}\right)$ is the horizontal component of $\xi=\left(\xi^{1}, \ldots, \xi^{r}\right) \in \Omega$. 
Proof We test the sub-elliptic system (1.1) with the testing function $\varphi=\phi^{2} v$ with $v=u-$ $u_{0}-\left(\xi^{1}-\xi_{0}^{1}\right) p_{0}$, where $\phi \in C_{0}^{\infty}\left(B_{R}\left(\xi_{0}\right), \mathbb{R}^{N}\right)$ is a cut-off function satisfying $0 \leq \phi \leq 1,|X \phi| \leq$ $\frac{C}{R-\rho}$, and $\phi \equiv 1$ on $B_{\rho}\left(\xi_{0}\right)$. This yields

$$
\begin{aligned}
\int_{B_{R}\left(\xi_{0}\right)} A_{i}^{\alpha}(\xi, u, X u) \phi^{2}\left(X u-p_{0}\right) d \xi= & -2 \int_{B_{R}\left(\xi_{0}\right)} \phi X \phi A_{i}^{\alpha}(\xi, u, X u) v d \xi \\
& +\int_{B_{R}\left(\xi_{0}\right)} B^{\alpha}(\xi, u, X u) \varphi^{\alpha} d \xi
\end{aligned}
$$

Adding this to the equations

$$
\begin{aligned}
-\int_{B_{R}\left(\xi_{0}\right)} A_{i}^{\alpha}\left(\xi, u, p_{0}\right) \phi^{2}\left(X u-p_{0}\right) d \xi= & 2 \int_{B_{R}\left(\xi_{0}\right)} \phi X \phi A_{i}^{\alpha}\left(\xi, u, p_{0}\right) v d \xi \\
& -\int_{B_{R}\left(\xi_{0}\right)} A_{i}^{\alpha}\left(\xi, u, p_{0}\right) X \varphi^{\alpha} d \xi
\end{aligned}
$$

and

$$
0=\int_{B_{R}\left(\xi_{0}\right)} A_{i}^{\alpha}\left(\xi_{0}, u_{0}, p_{0}\right) X \varphi^{\alpha}
$$

we have

$$
\begin{aligned}
& \int_{B_{R}\left(\xi_{0}\right)}\left[A_{i}^{\alpha}(\xi, u, X u)-A_{i}^{\alpha}\left(\xi, u, p_{0}\right)\right] \phi^{2}\left(X u-p_{0}\right) d \xi \\
& =2 \int_{B_{R}\left(\xi_{0}\right)}\left[A_{i}^{\alpha}\left(\xi, u, p_{0}\right)-A_{i}^{\alpha}(\xi, u, X u)\right] \phi v X \phi d \xi \\
& \quad+\int_{B_{R}\left(\xi_{0}\right)}\left[A_{i}^{\alpha}\left(\xi, u_{0}+\left(\xi^{1}-\xi_{0}^{1}\right) p_{0}, p_{0}\right)-A_{i}^{\alpha}\left(\xi, u, p_{0}\right)\right] X \varphi^{\alpha} d \xi \\
& \quad+\int_{B_{R}\left(\xi_{0}\right)}\left[A_{i}^{\alpha}\left(\xi_{0}, u_{0}, p_{0}\right)-A_{i}^{\alpha}\left(\xi, u_{0}+\left(\xi^{1}-\xi_{0}^{1}\right) p_{0}, p_{0}\right)\right] X \varphi^{\alpha} d \xi \\
& \quad+\int_{B_{R}\left(\xi_{0}\right)} B^{\alpha}(\xi, u, X u) \varphi^{\alpha} d \xi \\
& =: I+I I+I I I+I V+V,
\end{aligned}
$$

with the obvious labeling for $I-V$.

The left hand side of (3.8) can be estimated via the uniformly elliptic condition (H2) (also see (1.10)),

$$
\begin{aligned}
& \lambda_{0} \int_{B_{R}\left(\xi_{0}\right)}\left(\left|X u-p_{0}\right|^{2}+\left|X u-p_{0}\right|^{m}\right) \phi^{2} d \xi \\
& \quad \leq \int_{B_{R}\left(\xi_{0}\right)}\left[A_{i}^{\alpha}(\xi, u, X u)-A_{i}^{\alpha}\left(\xi, u, p_{0}\right)\right]\left(X u-p_{0}\right) \phi^{2} d \xi .
\end{aligned}
$$


For $\varepsilon>0$ to be fixed later we have, using the version (1.8) of (H1), and Young's inequality,

$$
\begin{aligned}
I \leq & 2 C(L) \int_{B_{R}\left(\xi_{0}\right)}\left(1+|X u|^{2}+\left|p_{0}\right|^{2}\right)^{\frac{m-2}{2}}\left|X u-p_{0}\right||\phi||v \| X \phi| d \xi \\
\leq & C \varepsilon \int_{B_{R}\left(\xi_{0}\right)}\left|X u-p_{0}\right|^{2}|\phi|^{2} d \xi+\frac{C}{\varepsilon(R-\rho)^{2}} \int_{B_{R}\left(\xi_{0}\right)}|v|^{2} d \xi \\
& +C \varepsilon \int_{B_{R}\left(\xi_{0}\right)}\left|X u-p_{0}\right|^{m}|\phi|^{\frac{m}{m-1}} d \xi+\frac{C}{\varepsilon^{m-1}(R-\rho)^{m}} \int_{B_{R}\left(\xi_{0}\right)}|v|^{m} d \xi
\end{aligned}
$$

Using Jensen's inequality, (2.6), and the fact that $\eta(t s) \leq t \eta(s)$ for $t \geq 1$, we arrive at

$$
\begin{aligned}
(1 & \left.+\left|p_{0}\right|\right)^{m} \kappa^{2}(\cdot) \int_{B_{R}\left(\xi_{0}\right)} \eta\left(|v|^{2}\right) d \xi \\
& \leq \omega_{G} R^{Q-2}\left[\left(1+\left|p_{0}\right|\right)^{m} \kappa^{2}(\cdot) R^{2}\right] \eta\left(f_{B_{R}\left(\xi_{0}\right)}|v|^{2} d \xi\right) \\
& \leq \omega_{G} R^{Q-2}\left[f_{B_{R}\left(\xi_{0}\right)}|v|^{2} d \xi+\left(1+\left|p_{0}\right|\right)^{m} \kappa^{2}(\cdot) R^{2} \eta\left(\left(1+\left|p_{0}\right|\right)^{m} \kappa^{2}(\cdot) R^{2}\right)\right] \\
& \leq R^{-2} \int_{B_{R}\left(\xi_{0}\right)}|v|^{2} d \xi+\omega_{G} R^{Q}\left(1+\left|p_{0}\right|\right)^{2 m} \kappa^{4}(\cdot) \eta\left(R^{2}\right),
\end{aligned}
$$

where $\kappa(\cdot)$ is abbreviation of the function $\kappa\left(\left|u_{0}\right|+\left|p_{0}\right|\right)$. Also note that (2.6) in the second last inequality is applied with the assumption $R<\rho_{1}^{m / 2}\left(\left|u_{0}\right|,\left|p_{0}\right|\right) \leq 1$.

Using the Dini continuity condition (H3), Young's inequality, and (3.11) in $I I$, we obtain

$$
\begin{aligned}
I I \leq & \left(1+\left|p_{0}\right|\right)^{\frac{m}{2}} \kappa\left(\left|u_{0}\right|+R\left|p_{0}\right|\right) \int_{B_{R}\left(\xi_{0}\right)} \eta^{\frac{1}{2}}\left(|v|^{2}\right)\left|X u-p_{0}\right| \phi^{2} d \xi \\
\leq & \varepsilon \int_{B_{R}\left(\xi_{0}\right)}\left|X u-p_{0}\right|^{2}|\phi|^{2} d \xi+\varepsilon^{-1}\left(1+\left|p_{0}\right|\right)^{m} \kappa^{2}(\cdot) \int_{B_{R}\left(\xi_{0}\right)} \eta\left(|v|^{2}\right) d \xi \\
& \leq \varepsilon \int_{B_{R}\left(\xi_{0}\right)}\left|X u-p_{0}\right|^{2}|\phi|^{2} d \xi+\frac{1}{\varepsilon(R-\rho)^{2}} \int_{B_{R}\left(\xi_{0}\right)}|v|^{2} d \xi \\
& +\varepsilon^{-1} \omega_{G} R^{Q}\left(1+\left|p_{0}\right|\right)^{2 m} \kappa^{4}(\cdot) \eta\left(R^{2}\right) .
\end{aligned}
$$

Similarly,

$$
\begin{aligned}
I I I & \leq 2\left(1+\left|p_{0}\right|\right)^{\frac{m}{2}} \kappa\left(\left|u_{0}\right|+R\left|p_{0}\right|\right) \int_{B_{R}\left(\xi_{0}\right)} \eta^{\frac{1}{2}}\left(|v|^{2}\right)|v||X \phi||\phi| d \xi \\
& \leq \frac{C}{(R-\rho)^{2}} \int_{B_{R}\left(\xi_{0}\right)}|v|^{2} d \xi+\left(1+\left|p_{0}\right|\right)^{m} \kappa^{2}(\cdot) \int_{B_{R}\left(\xi_{0}\right)} \eta\left(|v|^{2}\right) d \xi \\
& \leq \frac{C}{(R-\rho)^{2}} \int_{B_{R}\left(\xi_{0}\right)}|v|^{2} d \xi+\omega_{G} R^{Q}\left(1+\left|p_{0}\right|\right)^{2 m} \kappa^{4}(\cdot) \eta\left(R^{2}\right)
\end{aligned}
$$

and

$$
\begin{aligned}
I V \leq & \left(1+\left|p_{0}\right|\right)^{\frac{m}{2}} \kappa\left(\left|u_{0}\right|+R\left|p_{0}\right|\right) \\
& \times \int_{B_{R}\left(\xi_{0}\right)} \eta^{\frac{1}{2}}\left(R^{2}\left(1+\left|p_{0}\right|^{2}\right)\right)\left[\left|X u-p_{0}\right| \phi^{2}+2|\phi||v||X \phi|\right] d \xi
\end{aligned}
$$




$$
\begin{aligned}
\leq & \varepsilon \int_{B_{R}\left(\xi_{0}\right)}\left|X u-p_{0}\right|^{2}|\phi|^{2} d \xi+\frac{C \varepsilon}{(R-\rho)^{2}} \int_{B_{R}\left(\xi_{0}\right)}|v|^{2} d \xi \\
& +2 \varepsilon^{-1} \omega_{G} R^{Q}\left(1+\left|p_{0}\right|\right)^{m} \kappa^{2}(\cdot) \eta\left(f_{B_{R}\left(\xi_{0}\right)} R^{2}\left(1+\left|p_{0}\right|^{2}\right) d \xi\right) \\
\leq & \varepsilon \int_{B_{R}\left(\xi_{0}\right)}\left|X u-p_{0}\right|^{2}|\phi|^{2} d \xi+\frac{C \varepsilon}{(R-\rho)^{2}} \int_{B_{R}\left(\xi_{0}\right)}|v|^{2} d \xi \\
& +2 \varepsilon^{-1} \omega_{G} R^{Q}\left(1+\left|p_{0}\right|\right)^{m+2} \kappa^{4}(\cdot) \eta\left(R^{2}\right),
\end{aligned}
$$

where we have used $\kappa \geq 1$ in the last inequality.

Finally, the term $V$ can be estimated by using the controllable growth condition (H4), Hölder's inequality, and Young's inequality. This yields

$$
\begin{aligned}
V & \leq c \int_{B_{R}\left(\xi_{0}\right)}\left(1+|u|^{r-1}+|X u|^{m(1-1 / r)}\right) \varphi d \xi \\
& \leq c\left(\int_{B_{R}\left(\xi_{0}\right)}|\varphi|^{r} d \xi\right)^{1 / r}\left(\int_{B_{R}\left(\xi_{0}\right)}\left(1+|u|^{r}+|X u|^{m}\right) d \xi\right)^{(r-1) / r} \\
& \leq c\left(\int_{B_{R}\left(\xi_{0}\right)}|X \varphi|^{m} d \xi\right)^{1 / m}\left(\int_{B_{R}\left(\xi_{0}\right)}\left(1+|u|^{r}+|X u|^{m}\right) d \xi\right)^{(r-1) / r} \\
\leq & \varepsilon \int_{B_{R}\left(\xi_{0}\right)}|X \varphi|^{m} d \xi+C(\varepsilon)\left(\int_{B_{R}\left(\xi_{0}\right)}\left(1+|u|^{r}+|X u|^{m}\right) d \xi\right)^{m(r-1) / r(m-1)} \\
\leq & 2^{m-1} \varepsilon \int_{B_{R}\left(\xi_{0}\right)}\left|X u-p_{0}\right|^{m}|\phi|^{m} d \xi+\frac{2^{m-1} C \varepsilon}{(R-\rho)^{m}} \int_{B_{R}\left(\xi_{0}\right)}|v|^{m} d \xi \\
& +C(\varepsilon)\left(\int_{B_{R}\left(\xi_{0}\right)}\left(1+|u|^{r}+|X u|^{m}\right) d \xi\right)^{m(r-1) / r(m-1)},
\end{aligned}
$$

where we have used the fact $|X \varphi|^{m} \leq 2^{m-1}\left(\phi^{m}\left|X u-p_{0}\right|^{m}+\frac{C}{(R-\rho)^{m}}|v|^{m}\right)$.

Applying these estimates to (3.8), we obtain

$$
\begin{aligned}
& \Lambda \int_{B_{\rho}\left(\xi_{0}\right)}\left(\left|X u-p_{0}\right|^{2}+\left|X u-p_{0}\right|^{m}\right) \phi^{2} d \xi \\
& \quad \leq \frac{(C+1)\left(\varepsilon+\varepsilon^{-1}\right)}{(R-\rho)^{2}} \int_{B_{R}\left(\xi_{0}\right)}|v|^{2} d \xi+\frac{C\left(\varepsilon^{1-m}+2^{m-1} \varepsilon\right)}{(R-\rho)^{m}} \int_{B_{R}\left(\xi_{0}\right)}|v|^{m} d \xi \\
& \quad+\left(3 \varepsilon^{-1}+1\right) \omega_{G} R^{Q}\left(1+\left|p_{0}\right|\right)^{2 m} \kappa^{4}(\cdot) \eta\left(R^{2}\right) \\
& \quad+C(\varepsilon)\left(\int_{B_{R}\left(\xi_{0}\right)}\left(1+|u|^{r}+|X u|^{m}\right) d \xi\right)^{m(r-1) / r(m-1)}
\end{aligned}
$$

where $\Lambda=\lambda_{0}-\left(C+2+2^{m-1}\right) \varepsilon$. The conclusion follows by choosing suitable $\varepsilon$ such that $\Lambda>0$. We refer to [7] for a similar and detailed argument.

\section{Proof of main theorem}

In this section, we will complete the proof of the partial regularity results via the following lemmas. In the sequel, we always suppose that $u \in H W^{1, m}\left(\Omega, R^{N}\right)$ with $m>2$ is a weak solution to (1.1) with the assumptions of $(\mathrm{H} 1)-(\mathrm{H} 4)$, and $(\mu 1)-(\mu 3)$. First of all, we provide a linearization strategy for nonlinear sub-elliptic systems as in (1.1). 
Lemma 4 Let $B_{\rho}\left(\xi_{0}\right) \subset \subset \Omega$ with $\rho \leq \rho_{1}^{\frac{m}{2}}\left(\left|u_{0}\right|,\left|p_{0}\right|\right)$ and $\varphi \in C_{0}^{\infty}\left(B_{\rho}\left(\xi_{0}\right), R^{N}\right)$ satisfying $|\varphi| \leq \rho^{2}$ and $\sup _{B_{\rho}\left(\xi_{0}\right)}|X \varphi| \leq 1$. Then there exists a constant $C_{1} \geq 1$, such that

$$
\begin{aligned}
& f_{B_{\rho}\left(\xi_{0}\right)} A_{i, j_{\beta}^{j}}^{\alpha}\left(\xi_{0}, u_{0}, p_{0}\right)\left(X u-p_{0}\right) X \varphi^{\alpha} d \xi \\
& \leq C_{1}\left[\Phi\left(\xi_{0}, \rho, p_{0}\right)+\omega^{\frac{1}{m}}\left(\left|p_{0}\right|, \Phi\left(\xi_{0}, \rho, p_{0}\right)\right) \Phi^{\frac{1}{2}}\left(\xi_{0}, \rho, p_{0}\right)\right. \\
& \left.\quad+F\left(\left|u_{0}\right|,\left|p_{0}\right|\right) \eta^{\frac{1}{2}}\left(\rho^{2}\right)\right] \sup _{B_{\rho}\left(\xi_{0}\right)}|X \varphi|,
\end{aligned}
$$

where $F(s, t)=K_{1}(s, t)+(1+s+t)^{r-1}$ and $C_{1}=2^{\frac{m-2}{2}}\left(C(L)+2 c+4+2 C_{p}\right)>1$.

Proof We first write

$$
\left|A_{i}^{\alpha}\left(\xi_{0}, u_{0}, X u\right)-A_{i}^{\alpha}\left(\xi_{0}, u_{0}, p_{0}\right)\right|=\int_{0}^{1} A_{i, p_{\beta}^{j}}^{\alpha}\left(\xi_{0}, u_{0}, \theta X u+(1-\theta) p_{0}\right)\left(X u-p_{0}\right) d \theta
$$

Noting that $\int_{B_{\rho}\left(\xi_{0}\right)} A_{i}^{\alpha}\left(\xi_{0}, u_{0}, p_{0}\right) X \varphi^{\alpha} d \xi=0$ and using the weak form of $u$, we conclude

$$
\begin{aligned}
f_{B_{\rho}\left(\xi_{0}\right)} & A_{i, p_{\beta}^{\prime}}^{\alpha}\left(\xi_{0}, u_{0}, p_{0}\right)\left(X u-p_{0}\right) X \varphi^{\alpha} d \xi \\
\leq & f_{B_{\rho}\left(\xi_{0}\right)}\left[\int_{0}^{1}\left(A_{i, p_{\beta}^{j}}^{\alpha}\left(\xi_{0}, u_{0}, p_{0}\right)-A_{i, p_{\beta}^{j}}^{\alpha}\left(\xi_{0}, u_{0}, \theta X u+(1-\theta) p_{0}\right)\right)\left(X u-p_{0}\right) d \theta\right] d \xi \\
\quad & \times \sup _{B_{\rho}\left(\xi_{0}\right)}|X \varphi| \\
& +f_{B_{\rho}\left(\xi_{0}\right)}\left[A_{i}^{\alpha}\left(\xi_{0}, u_{0}, X u\right)-A_{i}^{\alpha}\left(\xi, u_{0}+p_{0}\left(\xi-\xi_{0}\right), X u\right)\right] \sup _{B_{\rho}\left(\xi_{0}\right)}|X \varphi| \\
& +f_{B_{\rho}\left(\xi_{0}\right)}\left[A_{i}^{\alpha}\left(\xi, u_{0}+p_{0}\left(\xi-\xi_{0}\right), X u\right)-A_{i}^{\alpha}(\xi, u, X u)\right] \sup _{B_{\rho}\left(\xi_{0}\right)}|X \varphi| \\
& +f_{B_{\rho}\left(\xi_{0}\right)} B^{\alpha}(\xi, u, X u) \varphi^{\alpha} d \xi \\
:= & I^{\prime}+I I^{\prime}+I I I^{\prime}+I V^{\prime}
\end{aligned}
$$

with the obvious meaning of $I-I V$. In order to estimate the term $I$, we use (1.4) and (1.9) to first get

$$
\begin{aligned}
\left|A_{i, p_{\beta}^{j}}^{\alpha}\left(\xi_{0}, u_{0}, p_{0}\right)-A_{i, p_{\beta}^{j}}^{\alpha}\left(\xi_{0}, u_{0}, \theta X u+(1-\theta) p_{0}\right)\right|^{\frac{1}{m}+\left(1-\frac{1}{m}\right)} \\
\leq\left[C\left(1+\left|p_{0}\right|^{2}+\left|\theta\left(X u-p_{0}\right)+p_{0}\right|^{2}\right)^{\frac{m-2}{2}} \omega\left(\left|p_{0}\right|,\left|\theta\left(X u-p_{0}\right)\right|^{2}\right)\right]^{\frac{1}{m}} \\
\quad \times\left[L\left(1+\left|p_{0}\right|^{2}\right)^{\frac{m-2}{2}}+L\left(1+\left|\theta\left(X u-p_{0}\right)+p_{0}\right|^{2}\right)^{\frac{m-2}{2}}\right]^{1-\frac{1}{m}} \\
\leq C(L)\left(1+\left|p_{0}\right|^{2}\right)^{\frac{m-2}{2}}\left|X u-p_{0}\right|^{m-2} \omega^{\frac{1}{m}}\left(\left|p_{0}\right|,\left|X u-p_{0}\right|^{2}\right) .
\end{aligned}
$$


Using (4.3), Hölder's inequality, the fact that $t \rightarrow \omega^{2}(s, t)$ is concave, and Jensen's inequality, we have

$$
\begin{aligned}
I^{\prime} & \leq C(L) f_{B_{\rho}\left(\xi_{0}\right)} \omega^{\frac{1}{m}}\left(\left|p_{0}\right|,\left|X u-p_{0}\right|^{2}\right)\left|X u-p_{0}\right|^{m-1} \sup _{B_{\rho}\left(\xi_{0}\right)}|X \varphi| d \xi \\
& \leq C(L) \sup _{B_{\rho}\left(\xi_{0}\right)}|X \varphi|\left[f_{B_{\rho}\left(\xi_{0}\right)} \omega\left(\left|p_{0}\right|,\left|X u-p_{0}\right|^{2}\right) d \xi\right]^{\frac{1}{m}}\left\{f_{B_{\rho}\left(\xi_{0}\right)}\left|X u-p_{0}\right|^{m} d \xi\right\}^{\frac{m-1}{m}} \\
& \leq C(L) \sup _{B_{\rho}\left(\xi_{0}\right)}|X \varphi| \omega^{\frac{1}{m}}\left(\left|p_{0}\right|, f_{B_{\rho}\left(\xi_{0}\right)}\left|X u-p_{0}\right|^{2} d \xi\right)\left\{f_{B_{\rho}\left(\xi_{0}\right)}\left|X u-p_{0}\right|^{m} d \xi\right\}^{\frac{m-1}{m}} \\
& \leq C(L) \omega^{\frac{1}{m}}\left(\left|p_{0}\right|, \Phi\left(\xi_{0}, \rho, p_{0}\right)\right) \Phi^{\frac{1}{2}}\left(\xi_{0}, \rho, p_{0}\right) \sup _{B_{\rho}\left(\xi_{0}\right)}|X \varphi|,
\end{aligned}
$$

where we have used the fact $\frac{m}{m-1}<2$ and the assumption $\Phi\left(\xi_{0}, \rho, p_{0}\right) \leq 1$.

The term $I I^{\prime}$ can be estimated using the Dini continuity condition (1.6) and the fact that $\eta(t s) \leq t \eta(s)$ for $t \geq 1$. We have

$$
\begin{aligned}
I I^{\prime} \leq & \sup _{B_{\rho}\left(\xi_{0}\right)}|X \varphi| \kappa(\cdot) \mu\left(\rho\left(1+\left|p_{0}\right|\right)\right) f_{B_{\rho}\left(\xi_{0}\right)}(1+|X u|)^{\frac{m}{2}} d \xi \\
\leq & \sup _{B_{\rho}\left(\xi_{0}\right)}|X \varphi| \kappa(\cdot)\left(1+\left|p_{0}\right|\right) \eta^{\frac{1}{2}}\left(\rho^{2}\right) f_{B_{\rho}\left(\xi_{0}\right)}\left(1+\left|p_{0}\right|+\left|X u-p_{0}\right|\right)^{\frac{m}{2}} d \xi \\
\leq & 2^{\frac{m-2}{2}} \sup _{B_{\rho}\left(\xi_{0}\right)}|X \varphi|\left[\kappa(\cdot)\left(1+\left|p_{0}\right|\right)^{1+\frac{m}{2}} \eta^{\frac{1}{2}}\left(\rho^{2}\right)+\kappa^{2}(\cdot)\left(1+\left|p_{0}\right|\right)^{2} \eta\left(\rho^{2}\right)\right. \\
& \left.+f_{B_{\rho}\left(\xi_{0}\right)}\left|X u-p_{0}\right|^{m} d \xi\right] \\
\leq & 2^{\frac{m-2}{2}}\left[\Phi\left(\xi_{0}, \rho, p_{0}\right)+2 \kappa^{2}(\cdot)\left(1+\left|p_{0}\right|\right)^{1+\frac{m}{2}} \eta^{\frac{1}{2}}\left(\rho^{2}\right)\right] \sup _{B_{\rho}\left(\xi_{0}\right)}|X \varphi|,
\end{aligned}
$$

where we have used the fact that $\eta\left(\rho^{2}\right) \leq \eta^{\frac{1}{2}}\left(\rho^{2}\right)$, which follows from the nondecreasing property of the function $\eta(t),(\eta 4)$, and our assumption $\rho \leq \rho_{1} \leq 1$.

Similarly, it follows that by using (1.6), (3.11), and the Poincaré inequality (2.4) in the special case $p=q=2$

$$
\begin{aligned}
I I I^{\prime} \leq & \sup _{B_{\rho}\left(\xi_{0}\right)}|X \varphi| f_{B_{\rho}\left(\xi_{0}\right)} \kappa(\cdot) \eta^{\frac{1}{2}}\left(|v|^{2}\right)(1+|X u|)^{\frac{m}{2}} d \xi \\
\leq & 2^{\frac{m-2}{2}} \sup _{B_{\rho}\left(\xi_{0}\right)}|X \varphi|\left[f_{B_{\rho}\left(\xi_{0}\right)}\left|X u-p_{0}\right|^{m} d \xi+\kappa^{2}(\cdot) f_{B_{\rho}\left(\xi_{0}\right)} \eta\left(|v|^{2}\right) d \xi\right. \\
& \left.+\kappa(\cdot)\left(1+\left|p_{0}\right|\right)^{\frac{m}{2}} f_{B_{\rho}\left(\xi_{0}\right)} \eta^{\frac{1}{2}}\left(|v|^{2}\right) d \xi\right] \\
\leq & 2^{\frac{m-2}{2}} \sup _{B_{\rho}\left(\xi_{0}\right)}|X \varphi|\left[\Phi\left(\xi_{0}, \rho, p_{0}\right)\right. \\
& \left.+2 \rho^{-2} f_{B_{\rho}\left(\xi_{0}\right)}|v|^{2} d \xi+\kappa^{4}(\cdot) \eta\left(\rho^{2}\right)+\kappa^{2}(\cdot)\left(1+\left|p_{0}\right|\right)^{m} \eta^{\frac{1}{2}}\left(\rho^{2}\right)\right] \\
\leq & 2^{\frac{m-2}{2}} \sup _{B_{\rho}\left(\xi_{0}\right)}|X \varphi|\left[\Phi\left(\xi_{0}, \rho, p_{0}\right)\right.
\end{aligned}
$$




$$
\begin{aligned}
& \left.+2 C_{p} f_{B_{\rho}\left(\xi_{0}\right)}\left|X u-p_{0}\right|^{2} d \xi+2 \kappa^{4}(\cdot)\left(1+\left|p_{0}\right|\right)^{m} \eta^{\frac{1}{2}}\left(\rho^{2}\right)\right] \\
\leq & 2^{\frac{m-2}{2}}\left[\left(1+2 C_{p}\right) \Phi\left(\xi_{0}, \rho, p_{0}\right)+2 \kappa^{4}(\cdot)\left(1+\left|p_{0}\right|\right)^{m} \eta^{\frac{1}{2}}\left(\rho^{2}\right)\right] \sup _{B_{\rho}\left(\xi_{0}\right)}|X \varphi| .
\end{aligned}
$$

Note that by the assumption $\sup _{B_{\rho}\left(\xi_{0}\right)}|\varphi| \leq \rho^{2} \leq 1$ and $(\eta 4)$, we have

$$
\begin{aligned}
I V^{\prime} \leq & c f_{B_{\rho}\left(\xi_{0}\right)}\left(1+|u|^{r-1}+|X u|^{m(1-1 / r)}\right)|\varphi| d \xi \\
\leq & c f_{B_{\rho}\left(\xi_{0}\right)}|X u|^{m(1-1 / r)}|\varphi| d \xi+c f_{B_{\rho}\left(\xi_{0}\right)}\left|u-u_{0}-p_{0}\left(\xi^{1}-\xi_{0}^{1}\right)\right|^{r-1}|\varphi| d \xi \\
& +c \rho^{2}\left[1+\left(\left|u_{0}\right|+\rho\left|p_{0}\right|\right)^{r-1}\right] \\
\leq & c\left(f_{B_{\rho}\left(\xi_{0}\right)}\left|X u-p_{0}\right|^{m} d \xi\right)^{\frac{r-1}{r}}\left(f_{B_{\rho}\left(\xi_{0}\right)}|\varphi|^{r} d \xi\right)^{\frac{1}{r}} \\
& +c\left(f_{B_{\rho}\left(\xi_{0}\right)}\left|p_{0}\right|^{m} d \xi\right)^{\frac{r-1}{r}}\left(f_{B_{\rho}\left(\xi_{0}\right)}|\varphi|^{r} d \xi\right)^{\frac{1}{r}} \\
& +c\left(f_{B_{\rho}\left(\xi_{0}\right)}\left|X u-p_{0}\right|^{m} d \xi\right)^{\frac{r-1}{m}}\left(f_{B_{\rho}\left(\xi_{0}\right)}|\varphi|^{r} d \xi\right)^{\frac{1}{r}}+c \rho^{2}\left[1+\left(\left|u_{0}\right|+\left|p_{0}\right|\right)^{r-1}\right] \\
\leq & 2 c\left(f_{B_{\rho}\left(\xi_{0}\right)}\left|X u-p_{0}\right|^{m} d \xi\right)^{\frac{r-1}{r}}\left(f_{B_{\rho}\left(\xi_{0}\right)}|\varphi|^{r} d \xi\right)^{\frac{1}{r}} \\
& +c \rho^{2}\left[1+\left(\left|u_{0}\right|+\left|p_{0}\right|\right)^{r-1}+\left|p_{0}\right|^{m\left(1-\frac{1}{r}\right)}\right] \\
\leq & 2 c \Phi\left(\xi_{0}, \rho, p_{0}\right)+2 c \eta^{\frac{1}{2}}\left(\rho^{2}\right)\left[\left(1+\left|u_{0}\right|+\left|p_{0}\right|\right)^{r-1}\right]
\end{aligned}
$$

where we have used $r-1 \geq m(1-1 / r)$ and $\eta(s) \leq 1$ for $s \in(0,1]$.

Combining the estimates (4.4)-(4.7) with (4.2), we obtain the conclusion with $C_{1}=$ $2^{\frac{m-2}{2}}\left(C(L)+2 c+4+2 C_{p}\right)$.

The following lemma is to establish the excess improvement of the functional $\Phi$ as in (1.11). The strategy of the proof is to approximate the given solution by $A$-harmonic functions, for which suitable decay estimates are available from the classical theory.

Lemma 5 Assume that the conditions of Lemma 2 and the following smallness conditions hold:

$$
\begin{aligned}
& \omega^{\frac{1}{m}}\left(\left|u_{\xi_{0}, \rho}\right|+\left|(X u)_{\xi_{0}, \rho}\right|, \Phi\left(\xi_{0}, \rho,(X u)_{\xi_{0}, \rho}\right)\right)+\Phi^{1 / 2}\left(\xi_{0}, \rho,(X u)_{\xi_{0}, \rho}\right) \leq \frac{\delta}{2}, \\
& C_{2} F^{2}\left(\left|u_{\xi_{0}, \rho}\right|,\left|(X u)_{\xi_{0}, \rho}\right|\right) \eta\left(\rho^{2}\right) \leq \delta^{2}
\end{aligned}
$$

with $C_{2}=8 C_{1}^{2} C_{4}$, together with the radius condition

$$
\rho \leq \rho_{1}^{m / 2}\left(1+\left|u_{\xi_{0}, \rho}\right|, 1+\left|(X u)_{\xi_{0}, \rho}\right|\right) .
$$


Then we have the following excess improvement estimate with $\tau \in[\gamma, 1)$ :

$$
\Phi\left(\xi_{0}, \theta \rho\right) \leq \theta^{2 \tau} \Phi\left(\xi_{0}, \rho\right)+K^{*}\left(\left|u_{\xi_{0}, \rho}\right|,\left|(X u)_{\xi_{0}, \rho}\right|\right) \eta\left(\rho^{\frac{m}{m-1}}\right),
$$

where we have abbreviated $\Phi\left(\xi_{0}, r\right)=\Phi\left(\xi_{0}, r,(X u)_{\xi_{0}, r}\right)$, and $K^{*}(s, t)=C_{7} F^{2}(1+s, 1+t)$.

Proof We define $w=\left[u-u_{\xi_{0}, \rho}-(X u)_{\xi_{0}, \rho}\left(\xi^{1}-\xi_{0}^{1}\right)\right] \sigma^{-1}$, where

$$
\sigma=C_{1} \sqrt{\Phi\left(\xi_{0}, \rho\right)+4 \delta^{-2} F^{2}\left(\left|u_{\xi_{0}, \rho}\right|,\left|(X u)_{\xi_{0}, \rho}\right|\right) \eta\left(\rho^{2}\right)}
$$

with $C_{1}>1$ in Lemma 4 . Then we have $X w=\sigma^{-1}\left(X u-(X u)_{\xi_{0}, \rho}\right)$. Now we consider $B_{\rho}\left(\xi_{0}\right) \subset \subset \Omega$ such that $\rho \leq \rho_{1}^{\frac{m}{2}}\left(\left|u_{\xi_{0}, \rho}\right|,\left|(X u)_{\xi_{0}, \rho}\right|\right) \leq 1$. It yields

$$
f_{B_{\rho}\left(\xi_{0}\right)}|X w|^{2} d \xi=\sigma^{-2} \Phi\left(\xi_{0}, \rho\right) \leq \frac{1}{C_{1}^{2}} \leq 1
$$

Applying Lemma 4 on $B_{\rho}\left(\xi_{0}\right)$ to $u$, we have, for any $\varphi \in C_{0}^{\infty}\left(B_{\rho}\left(\xi_{0}\right), R^{N}\right)$,

$$
\begin{aligned}
& f_{B_{\rho}\left(\xi_{0}\right)} A_{i, p_{\beta}^{j}}^{\alpha}\left(\xi_{0}, u_{\xi_{0}, \rho},(X u)_{\xi_{0}, \rho}\right) X w X \varphi d \xi \\
& \quad \leq\left[\Phi^{1 / 2}\left(\xi_{0}, \rho\right)+\omega^{\frac{1}{m}}\left(\left|(X u)_{\xi_{0}, \rho}\right|, \Phi\left(\xi_{0}, \rho\right)\right)+\frac{\delta}{2}\right] \sup _{B_{\rho}\left(\xi_{0}\right)}|X \varphi| .
\end{aligned}
$$

In consideration of the smallness condition (4.8), we see that (4.12) and (4.13) imply the conditions (3.2) and (3.3) in Lemma 2, respectively. Also note that assumptions (H1) and (H2) with $u=u_{\xi_{0}, \rho}$, and $p=(X u)_{\xi_{0}, \rho}$, imply the conditions (3.1). So there exists a $A_{i, p_{\beta}^{j}}^{\alpha}\left(\xi_{0}, u_{\xi_{0}, \rho},(X u)_{\xi_{0}, \rho}\right)$-harmonic function $h \in H W^{1,2}\left(B_{\rho}\left(\xi_{0}\right), R^{N}\right)$ such that

$$
f_{B_{\rho}\left(\xi_{0}\right)}|X h|^{2} d \xi \leq 1
$$

and

$$
\rho^{-2} f_{B_{\rho}\left(\xi_{0}\right)}|w-h|^{2} d \xi \leq \varepsilon
$$

Using Lemma 3 on the ball $B_{2 \theta \rho}\left(\xi_{0}\right)$ with $u_{0}=u_{\xi_{0}, 2 \theta \rho}, \theta \in(0,1 / 4]$, and replacing $p_{0}$ by $(X u)_{\xi_{0}, \rho}+\sigma(X h)_{\xi_{0}, 2 \theta \rho}$, we obtain

$$
\begin{gathered}
\int_{B_{\theta \rho}\left(\xi_{0}\right)}\left(\left|X u-(X u)_{\xi_{0}, \rho}-\sigma(X h)_{\xi_{0}, 2 \theta \rho}\right|^{2}+\left|X u-(X u)_{\xi_{0}, \rho}-\sigma(X h)_{\xi_{0}, 2 \theta \rho}\right|^{m}\right) d \xi \\
\leq \frac{C_{c}}{(\theta \rho)^{2}} \int_{B_{2 \theta \rho}\left(\xi_{0}\right)}\left|u-u_{\xi_{0}, 2 \theta \rho}-\left((X u)_{\xi_{0}, \rho}+\sigma(X h)_{\xi_{0}, 2 \theta \rho}\right)\left(\xi^{1}-\xi_{0}^{1}\right)\right|^{2} d \xi \\
\quad+\frac{C_{c}}{(\theta \rho)^{m}} \int_{B_{2 \theta \rho}\left(\xi_{0}\right)}\left|u-u_{\xi_{0}, 2 \theta \rho}-\left((X u)_{\xi_{0}, \rho}+\sigma(X h)_{\xi_{0}, 2 \theta \rho}\right)\left(\xi^{1}-\xi_{0}^{1}\right)\right|^{m} d \xi \\
\quad+C_{c} \omega_{G}(2 \theta \rho)^{Q}\left[K_{1}\left(\left|u_{\xi_{0}, 2 \theta \rho}\right|,\left|(X u)_{\xi_{0}, \rho}+\sigma(X h)_{\xi_{0}, 2 \theta \rho}\right|\right) \eta\left((2 \theta \rho)^{2}\right)\right]
\end{gathered}
$$




$$
\begin{aligned}
& +C_{c}\left[\int_{B_{2 \theta_{\rho}}\left(\xi_{0}\right)}\left(1+|u|^{r}+|X u|^{m}\right) d \xi\right]^{m(r-1) / r(m-1)} \\
:= & I^{\prime \prime}+I I^{\prime \prime}+I I I^{\prime \prime}+I V^{\prime \prime} .
\end{aligned}
$$

Note that the smallness conditions (4.8)-(4.9) imply $\sigma^{2} C_{4}\left(=C_{1}^{2} C_{4} \Phi+\frac{1}{2} C_{2} F^{2} \eta \delta^{-2}\right) \leq 1$ with $C_{4}=\max \left\{C_{0},(2 \theta)^{-Q}\right\}$, where we have assumed $\frac{1}{2} C_{1}^{2} C_{4} \delta^{2} \leq 1$, which is no restriction. Then it follows by applying the prior estimate (3.5) for the $\mathcal{A}$-harmonic function $h$

$$
\left|\sigma(X h)_{\xi_{0}, 2 \theta \rho}\right| \leq \sigma \sup _{B_{2 \theta_{\rho}}\left(\xi_{0}\right)}|X h| \leq \sigma \sqrt{C_{0}}\left(f_{B_{\rho}\left(\xi_{0}\right)}|X h|^{2} d \xi\right)^{\frac{1}{2}} \leq \sigma \sqrt{C_{0}} \leq 1 .
$$

Furthermore, it follows by Poincaré inequality (2.4)

$$
\begin{aligned}
\left|u_{\xi_{0}, 2 \theta \rho}\right| & \leq\left|u_{\xi_{0}, \rho}\right|+\left|u_{\xi_{0}, 2 \theta \rho}-u_{\xi_{0}, \rho}\right| \\
& \leq\left|u_{\xi_{0}, \rho}\right|+(2 \theta)^{-Q / 2}\left(f_{B_{\rho}\left(\xi_{0}\right)}\left|u-(X u)_{\xi_{0}, \rho}\left(\xi^{1}-\xi_{0}^{1}\right)-u_{\xi_{0}, \rho}\right|^{2} d \xi\right)^{1 / 2} \\
& \leq\left|u_{\xi_{0}, \rho}\right|+(2 \theta)^{-Q / 2} \rho C_{p} \Phi^{1 / 2}\left(\xi_{0}, \rho\right) \\
& \leq\left|u_{\xi_{0}, \rho}\right|+\frac{\sigma C_{p}}{C_{1}(2 \theta)^{\frac{Q}{2}}} \\
& \leq\left|u_{\xi_{0}, \rho}\right|+\sigma \sqrt{C_{4}} \leq\left|u_{\xi_{0}, \rho}\right|+1
\end{aligned}
$$

where we have used the definition of $\sigma$ (4.11) and the fact $C_{1}>C_{p}$.

Recall that $g(\tau)=\int_{B_{2 \theta \rho}\left(\xi_{0}\right)}(u-\tau)^{2} d \xi$ has a minimal value at $\tau=u_{\xi_{0}, 2 \theta \rho}$. Noting that $u-$ $\left((X u)_{\xi_{0}, \rho}+\sigma(X h)_{\xi_{0}, 2 \theta \rho}\right)\left(\xi^{1}-\xi_{0}^{1}\right)$ has mean value $u_{\xi_{0}, 2 \theta \rho}$ on the ball $B_{2 \theta \rho}\left(\xi_{0}\right)$, and using the definition of $w$, and Poincaré inequality (2.4), we have

$$
\begin{aligned}
& \frac{1}{(\theta \rho)^{2}} f_{B_{2 \theta \rho}\left(\xi_{0}\right)}\left|u-u_{\xi_{0}, 2 \theta \rho}-\left((X u)_{\xi_{0}, \rho}+\sigma(X h)_{\xi_{0}, 2 \theta \rho}\right)\left(\xi^{1}-\xi_{0}^{1}\right)\right|^{2} d \xi \\
& \quad \leq \frac{4 \sigma^{2}}{(2 \theta \rho)^{2}}\left[f_{B_{2 \theta \rho}\left(\xi_{0}\right)}|w-h|^{2} d \xi+f_{B_{2 \theta_{\rho}}\left(\xi_{0}\right)}\left|h-h_{\xi_{0}, 2 \theta \rho}-(X h)_{\xi_{0}, 2 \theta \rho}\left(\xi^{1}-\xi_{0}^{1}\right)\right|^{2} d \xi\right] \\
& \quad \leq 4 \sigma^{2}\left[(2 \theta)^{-Q-2} \varepsilon+C_{p}^{2}(2 \theta \rho)^{2} f_{B_{2 \theta \rho}\left(\xi_{0}\right)}\left|X^{2} h\right|^{2} d \xi\right] \\
& \quad \leq C_{3}\left(\theta^{-Q-2} \varepsilon+\theta^{2}\right)\left[\Phi\left(\xi_{0}, \rho\right)+4 \delta^{-2} F^{2}\left(\left|u_{\xi_{0}, \rho}\right|,\left|(X u)_{\xi_{0}, \rho}\right|\right) \eta\left(\rho^{2}\right)\right]
\end{aligned}
$$

where $C_{3}=C_{1}^{2}\left(2^{-Q}+16 C_{p}^{2} C_{0}\right) \geq 1$, and in the last inequality we have used the definition of $\sigma$ (4.11) with $u_{0}=u_{\xi_{0}, 2 \theta \rho}$, and the fact that

$$
f_{B_{2 \theta_{\rho}\left(\xi_{0}\right)}}\left|X^{2} h\right| d \xi \leq \sup _{B_{\rho}\left(\xi_{0}\right)}\left|X^{2} h\right| \leq C_{0} \rho^{-2} f_{B_{\rho}\left(\xi_{0}\right)}|X h|^{2} d \xi \leq C_{0} \rho^{-2}
$$

Then it follows

$$
I^{\prime \prime} \leq C_{3} C_{c} \omega_{G}(2 \theta \rho)^{Q}\left(\theta^{-Q-2} \varepsilon+\theta^{2}\right)\left[\Phi\left(\xi_{0}, \rho\right)+4 \delta^{-2} F^{2}\left(\left|u_{\xi_{0}, \rho}\right|,\left|(X u)_{\xi_{0}, \rho}\right|\right) \eta\left(\rho^{2}\right)\right] .
$$


For $2<m<Q$, we have $\frac{Q-m}{Q m}=\frac{1}{m^{*}}<\frac{1}{m}<\frac{1}{2}$. Then there exists $t \in(0,1)$ such that $\frac{1}{m}=\frac{1}{2}(1-$ $t)+\frac{1}{m^{*}} t$. Using (2.5), (2.4), Young's inequality, and (4.19) in turn, we have

$$
\begin{aligned}
& f_{B_{2 \theta \rho}\left(\xi_{0}\right)}\left|u-u_{\xi_{0}, 2 \theta \rho}-\left((X u)_{\xi_{0}, \rho}+\sigma(X h)_{\xi_{0}, 2 \theta \rho}\right)\left(\xi^{1}-\xi_{0}^{1}\right)\right|^{m} d \xi \\
& \leq {\left[f_{B_{2 \theta \rho}\left(\xi_{0}\right)}\left|u-u_{\xi_{0}, 2 \theta \rho}-\left((X u)_{\xi_{0}, \rho}+\sigma(X h)_{\xi_{0}, 2 \theta \rho}\right)\left(\xi^{1}-\xi_{0}^{1}\right)\right|^{2} d \xi\right]^{(1-t) \frac{m}{2}} } \\
& \times\left[f_{B_{2 \theta \rho}\left(\xi_{0}\right)}\left|u-u_{\xi_{0}, 2 \theta \rho}-\left((X u)_{\xi_{0}, \rho}+\sigma(X h)_{\xi_{0}, 2 \theta \rho}\right)\left(\xi^{1}-\xi_{0}^{1}\right)\right|^{m^{*}} d \xi\right]^{t \frac{m}{m^{*}}} \\
& \leq\left\{(\theta \rho)^{2} C_{3}\left[\theta^{-Q-2} \varepsilon+\theta^{2}\right]\left[\Phi\left(\xi_{0}, \rho\right)+4 \delta^{-2} F^{2}\left(\left|u_{\xi_{0}, \rho}\right|,\left|(X u)_{\xi_{0}, \rho}\right|\right) \eta\left(\rho^{2}\right)\right]\right\}^{(1-t) \frac{m}{2}} \\
& \times\left(C_{p}^{m}(2 \theta \rho)^{m} f_{B_{2 \theta \rho}\left(\xi_{0}\right)}\left|X u-\left((X u)_{\xi_{0}, \rho}+\sigma(X h)_{\xi_{0}, 2 \theta \rho}\right)\right|^{m} d \xi\right)^{t} \\
& \leq\left(\varepsilon^{-t}\left(2 C_{p}\right)^{m t}\right)^{\frac{1}{1-t}}\left\{(\theta \rho)^{2} C_{3}\left[\theta^{-Q-2} \varepsilon+\theta^{2}\right]\right. \\
&\left.\times\left[\Phi\left(\xi_{0}, \rho\right)+4 \delta^{-2} F^{2}\left(\left|u_{\xi_{0}, \rho}\right|,\left|(X u)_{\xi_{0}, \rho}\right|\right) \eta\left(\rho^{2}\right)\right]\right\}^{\frac{m}{2}} \\
&+\varepsilon(\theta \rho)^{m} f_{B_{2 \theta \rho}\left(\xi_{0}\right)}\left|X u-\left((X u)_{\xi_{0}, \rho}+\sigma(X h)_{\xi_{0}, 2 \theta \rho}\right)\right|^{m} d \xi \\
& \leq\left(\varepsilon^{-t}\left(2 C_{p}\right)^{m t}\right)^{\frac{1}{1-t}}\left(C_{3}\right)^{\frac{m}{2}}(\theta \rho)^{m}\left[\theta^{-Q-2} \varepsilon+\theta^{2}\right] \\
& \times\left[\Phi\left(\xi_{0}, \rho\right)+4 \delta^{-2} F^{2}\left(\left|u_{\xi_{0}, \rho}\right|,\left|(X u)_{\xi_{0}, \rho}\right|\right) \eta\left(\rho^{2}\right)\right] \\
&+\varepsilon(\theta \rho)^{m} f_{B_{2 \theta \rho}\left(\xi_{0}\right)}\left|X u-\left((X u)_{\xi_{0}, \rho}+\sigma(X h)_{\xi_{0}, 2 \theta \rho}\right)\right|^{m} d \xi,
\end{aligned}
$$

here we have used the assumption $\sigma^{2} C_{4} \leq 1$. Furthermore, we obtain the estimate for the term $I I^{\prime \prime}$,

$$
\begin{aligned}
I I^{\prime \prime} \leq & C_{c} \omega_{G}(2 \theta \rho)^{Q}\left(\varepsilon^{-t}\left(2 C_{p}\right)^{m t}\right)^{\frac{1}{1-t}}\left(C_{3}\right)^{\frac{m}{2}}\left[\theta^{-Q-2} \varepsilon+\theta^{2}\right] \\
& \times\left[\Phi\left(\xi_{0}, \rho\right)+4 \delta^{-2} F^{2}\left(\left|u_{\xi_{0}, \rho}\right|,\left|(X u)_{\xi_{0}, \rho}\right|\right) \eta\left(\rho^{2}\right)\right] \\
& +\varepsilon C_{c} \int_{B_{2 \theta_{\rho}}\left(\xi_{0}\right)}\left|X u-\left((X u)_{\xi_{0}, \rho}+\sigma(X h)_{\xi_{0}, 2 \theta \rho}\right)\right|^{m} d \xi .
\end{aligned}
$$

Also, (4.17) and (4.18) yield

$$
\begin{aligned}
I I I^{\prime \prime} & \leq C_{c} \omega_{G}(2 \theta \rho)^{Q}\left[K_{1}\left(1+\left|u_{\xi_{0}, \rho}\right|, 1+\left|(X u)_{\xi_{0}, \rho}\right|\right) \eta\left((2 \theta \rho)^{2}\right)\right] \\
& \leq C_{c} \omega_{G}(2 \theta \rho)^{Q} F\left(1+\left|u_{\xi_{0}, \rho}\right|, 1+\left|(X u)_{\xi_{0}, \rho}\right|\right) \eta\left((2 \theta \rho)^{2}\right) .
\end{aligned}
$$

Using the Poincaré type inequality, we have

$$
\begin{aligned}
& {\left[f_{B_{2 \theta_{\rho}\left(\xi_{0}\right)}}\left(|X u|^{m}+|u|^{r}+1\right) d \xi\right]^{\frac{m(r-1)}{r(m-1)}}} \\
& \quad \leq\left[2^{m-1} f_{B_{2 \theta_{\rho}\left(\xi_{0}\right)}}\left(\left|X u-(X u)_{\xi_{0}, \rho}\right|^{m}\right) d \xi\right]^{\frac{m(r-1)}{r(m-1)}}+\left(2^{m-1}\left|(X u)_{\xi_{0}, \rho}\right|^{m}\right)^{\frac{m(r-1)}{r(m-1)}}
\end{aligned}
$$




$$
\begin{aligned}
& +\left[f_{B_{2 \theta_{\rho}}\left(\xi_{0}\right)} 2^{r-1}\left(\left|u-u_{\xi_{0}, \rho}-(X u)_{\xi_{0}, \rho}\left(\xi^{1}-\xi_{0}^{1}\right)\right|^{r}\right) d \xi\right]^{\frac{m(r-1)}{r(m-1)}} \\
& +\left[\left(1+2^{r-1}\left|u_{\xi_{0}, \rho}+(X u)_{\xi_{0}, \rho}\left(\xi^{1}-\xi_{0}^{1}\right)\right|^{r}\right)\right]^{\frac{m(r-1)}{r(m-1)}} \\
& \leq C\left[(2 \theta)^{-Q} f_{B_{\rho}\left(\xi_{0}\right)}\left(\left|X u-(X u)_{\xi_{0}, \rho}\right|^{m}\right) d \xi\right]^{\frac{m(r-1)}{r(m-1)}}+\left(2^{m-1}\left|(X u)_{\xi_{0}, \rho}\right|^{m}\right)^{\frac{m(r-1)}{r(m-1)}} \\
& +C\left[(2 \theta)^{-Q} f_{B_{\rho}\left(\xi_{0}\right)}\left|X u-(X u)_{\xi_{0}, \rho}\right|^{m} d \xi\right]^{\frac{r-1}{m-1}}+\left[\left(1+2^{r-1}\left|u_{\xi_{0}, \rho}+(X u)_{\xi_{0}, \rho}\right|^{r}\right)\right]^{\frac{m(r-1)}{r(m-1)}} \\
& \leq C\left[(2 \theta)^{-Q} \Phi\left(\xi_{0}, \rho\right)\right]^{\frac{m(r-1)}{r(m-1)}}+C\left(1+\left|u_{\xi_{0}, \rho}\right|+\left|(X u)_{\xi_{0}, \rho}\right|\right)^{\frac{m(r-1)}{r(m-1)}} \\
& \leq C\left(2+\left|u_{\xi_{0}, \rho}\right|+\left|(X u)_{\xi_{0}, \rho}\right|\right)^{\frac{m(r-1)}{r(m-1)}},
\end{aligned}
$$

where in the last inequality we have used the fact $(2 \theta)^{-Q} \Phi\left(\xi_{0}, \rho\right) \leq 1$, implied by the assumption $\sigma^{2} C_{4} \leq 1$ with $C_{4}=\max \left\{C_{0},(2 \theta)^{-Q}\right\}$. In view of $\frac{m(r-1)}{m-1}+\frac{m^{2}}{m-1}\left(1-\frac{1}{r}\right)<\frac{2 m(r-1)}{m-1}$, we have

$$
I V^{\prime \prime} \leq C \omega_{G}(2 \theta \rho)^{Q} F^{\frac{m}{r(m-1)}}\left(1+\left|u_{\xi_{0}, \rho}\right|,\left|(X u)_{\xi_{0}, \rho}\right|\right) \eta\left((2 \theta \rho)^{\frac{m}{m-1}}\right),
$$

where we have used $\frac{Q m(r-1)}{r(m-1)} \leq Q+\frac{m}{m-1}$ and $(2 \theta \rho)^{\frac{m}{m-1}} \leq \eta\left((2 \theta \rho)^{\frac{m}{m-1}}\right)$.

Joining the estimates of $I^{\prime \prime}, I I^{\prime \prime}, I I I^{\prime \prime}$, and $I V^{\prime \prime}$ with (4.16), we obtain

$$
\begin{aligned}
\int_{B_{\theta \rho}\left(\xi_{0}\right)} & \left|X u-(X u)_{\xi_{0}, \rho}-\sigma(X h)_{\xi_{0}, 2 \theta \rho}\right|^{2} d \xi \\
& +\left(1-C_{c} \varepsilon\right) \int_{B_{\theta \rho}\left(\xi_{0}\right)}\left|X u-(X u)_{\xi_{0}, \rho}-\sigma(X h)_{\xi_{0}, 2 \theta \rho}\right|^{m} d \xi \\
\leq & C_{c}\left(C_{3}\right)^{\frac{m}{2}} \omega_{G}(2 \theta \rho)^{Q}\left[\left(\left(2 C_{p}\right)^{m t} \varepsilon^{-t}\right)^{\frac{1}{1-t}}+1\right]\left[\theta^{-Q-2} \varepsilon+\theta^{2}\right] \\
& \times\left[\Phi\left(\xi_{0}, \rho\right)+4 \delta^{-2} F^{2}\left(\left|u_{\xi_{0}, \rho}\right|,\left|(X u)_{\xi_{0}, \rho}\right|\right) \eta\left(\rho^{2}\right)\right] \\
& +C_{c} \omega_{G}(2 \theta \rho)^{Q} F^{2}\left(\left|u_{\xi_{0}, \rho}\right|, 1+\left|(X u)_{\xi_{0}, \rho}\right|\right) \eta\left((2 \theta \rho)^{2}\right) \\
& +C \omega_{G}(2 \theta \rho)^{Q} F^{\frac{m}{r(m-1)}}\left(1+\left|u_{\xi_{0}, \rho}\right|,\left|(X u)_{\xi_{0}, \rho}\right|\right) \eta\left((2 \theta \rho)^{\frac{m}{m-1}}\right) \\
\leq & C_{5} \omega_{G}(2 \theta \rho)^{Q}\left[\theta^{-Q-2} \varepsilon+\theta^{2}\right]\left[\Phi\left(\xi_{0}, \rho\right)+4 \delta^{-2} F^{2}\left(\left|u_{\xi_{0}, \rho}\right|,\left|(X u)_{\xi_{0}, \rho}\right|\right) \eta\left(\rho^{2}\right)\right] \\
& +C \omega_{G}(2 \theta \rho)^{Q} F^{2}\left(1+\left|u_{\xi_{0}, \rho}\right|, 1+\left|(X u)_{\xi_{0}, \rho}\right|\right) \eta\left(\rho^{\frac{m}{m-1}}\right),
\end{aligned}
$$

where $C_{5}=C_{c}\left(C_{3}\right)^{\frac{m}{2}}\left[\left(\left(2 C_{p}\right)^{m t} \varepsilon^{-t}\right)^{\frac{1}{1-t}}+1\right]>1$.

We set $P=(X u)_{\xi_{0}, \rho}+\sigma(X h)_{\xi_{0}, 2 \theta \rho}$. Choosing a suitable small $\varepsilon>0$ such that $\left(1-C_{c} \varepsilon\right)>0$, and considering the smallness condition (4.10) (it implies $\rho \leq \rho_{1}\left(\left|u_{\xi_{0}, 2 \theta \rho}\right|,|P|\right)$, see (4.17) and (4.18) above), we deduce that

$$
\begin{aligned}
\Phi & \left(\xi_{0}, \theta \rho,(X u)_{\xi_{0}, \theta \rho}\right) \\
& =f_{B_{\theta \rho}\left(\xi_{0}\right)}\left(\left|X u-(X u)_{\xi_{0}, \theta \rho}\right|^{2}+\left|X u-(X u)_{\xi_{0}, \theta \rho}\right|^{m}\right) d \xi \\
& \leq f_{B_{\theta \rho}\left(\xi_{0}\right)}\left(|X u-P|^{2}+|X u-P|^{m}\right) d \xi
\end{aligned}
$$




$$
\begin{aligned}
\leq & C_{6}\left[\theta^{-Q-2} \varepsilon+\theta^{2}\right]\left[\Phi\left(\xi_{0}, \rho,(X u)_{\xi_{0}, \rho}\right)+4 \delta^{-2} \eta\left(\rho^{2}\right) F^{2}\left(\left|u_{\xi_{0}, \rho}\right|,\left|(X u)_{\xi_{0}, \rho}\right|\right)\right] \\
& +C_{6} F^{2}\left(1+\left|u_{\xi_{0}, \rho}\right|, 1+\left|(X u)_{\xi_{0}, \rho}\right|\right) \eta\left(\rho^{\frac{m}{m-1}}\right),
\end{aligned}
$$

where $C_{6}=\frac{C_{5} 2^{Q}}{\left[1-C_{c} \varepsilon\right]}>1$.

For a given $\tau \in[\gamma, 1)$, we now specify $\varepsilon=\theta^{Q+4}, \theta \in(0,1 / 4]$ such that $2 C_{6} \theta^{2} \leq \theta^{2 \tau}$. Then we have

$$
\begin{aligned}
\Phi\left(\xi_{0}, \theta \rho\right) & \leq \theta^{2 \tau} \Phi\left(\xi_{0}, \rho\right)+\left(8 C_{6} \theta^{2} \delta^{-2}+C_{6}\right) F^{2}\left(1+\left|u_{\xi_{0}, \rho}\right|, 1+\left|(X u)_{\xi_{0}, \rho}\right|\right) \eta\left(\rho^{\frac{m}{m-1}}\right) \\
& \leq \theta^{2 \tau} \Phi\left(\xi_{0}, \rho\right)+C_{7} F^{2}\left(1+\left|u_{\xi_{0}, \rho}\right|, 1+\left|(X u)_{\xi_{0}, \rho}\right|\right) \eta\left(\rho^{\frac{m}{m-1}}\right) \\
& :=\theta^{2 \tau} \Phi\left(\xi_{0}, \rho\right)+K^{*}\left(\left|u_{\xi_{0}, \rho}\right|,\left|(X u)_{\xi_{0}, \rho}\right|\right) \eta\left(\rho^{\frac{m}{m-1}}\right),
\end{aligned}
$$

where $C_{7}=8 C_{6} \theta^{2} \delta^{-2}+C_{6}>1$ and $K^{*}(s, t)=C_{7} F^{2}(1+s, 1+t)$. Then the proof of Lemma 5 is complete.

For $T>0$, we find $\Phi_{0}(T)>0$ (depending on $Q, N, \lambda, L, \tau$, and $\omega$ ) such that

$$
\begin{aligned}
& \omega^{\frac{1}{m}}\left(2 T, 2 \Phi_{0}(T)\right)+2 \Phi_{0}^{\frac{1}{2}}(T) \leq \frac{1}{2} \delta \text { and } \\
& 2\left(1+\sqrt{C_{p}}\right) \sqrt{\Phi_{0}(T)} \leq \theta^{Q / 2}\left(1-\theta^{\tau}\right) T .
\end{aligned}
$$

With $\Phi_{0}(T)$ from (4.26) and (4.27), we choose $\rho_{0}(T) \in(0,1]$ (depending on $Q, N, \lambda, L, \tau$, $\omega, \eta$, and $\kappa)$ such that

$$
\begin{aligned}
& \rho_{0}(T) \leq \rho_{1}^{m / 2}(1+2 T, 1+2 T), \\
& C_{2} F^{2}(2 T, 2 T) \eta\left(\rho_{0}(T)^{2}\right) \leq \delta^{2}, \\
& K_{0}(T) \eta\left(\rho_{0}(T)^{2}\right) \leq\left(\theta^{2 \gamma}-\theta^{2 \tau}\right) \Phi_{0}(T) \text { and } \\
& 2\left(1+C_{p}\right) K_{0}(T) H\left(\rho_{0}(T)^{2}\right) \leq \theta^{Q}\left(1-\theta^{\gamma}\right)^{2}\left(\theta^{2 \gamma}-\theta^{2 \tau}\right) T^{2},
\end{aligned}
$$

where $K_{0}(T):=K^{*}(2 T, 2 T)$.

By the proof method of Lemma 5.1 in [12] and conditions (4.26)-(4.31), Lemma 6 can be proved. As we know, it is sufficient to complete the proof of Theorem 1 once we obtain Lemma 6.

Lemma 6 Assume that for some $T_{0}>0$ and $B_{\rho}\left(\xi_{0}\right) \subset \subset \Omega$ we have

(1) $\left|u_{\xi_{0}, \rho}\right|+\left|(X u)_{\xi_{0}, \rho}\right| \leq T_{0}$;

(2) $\rho \leq \rho_{0}\left(T_{0}\right)$;

(3) $\Phi\left(\xi_{0}, \rho\right) \leq \Phi_{0}\left(T_{0}\right)$.

Then the smallness conditions (4.8)-(4.10) are satisfied on the balls $B_{\theta^{j}}\left(\xi_{0}\right)$ for $j \in \mathrm{N} \cup\{0\}$. Moreover, the limit $\Lambda_{\xi_{0}}=\lim _{j \rightarrow \infty}(X u)_{\xi_{0}, \theta^{j} \rho}$ exists, and the estimate

$$
f_{B_{\rho}\left(\xi_{0}\right)}\left|X u-\Lambda_{\xi_{0}}\right|^{2} d \xi \leq C_{8}\left(\left(\frac{r}{\rho}\right)^{2 \tau} \Phi\left(\xi_{0}, \rho\right)+H\left(r^{2}\right)\right)
$$

holds for $0<r \leq \rho$ with a constant $C_{8}=C_{8}\left(Q, N, \lambda, L, \tau, T_{0}\right)$. 
Proof The proof is very similar to the proof of Lemma 5.1 in [12]. We omit it here.

Competing interests

The authors declare that there is no competing interests regarding the publication of this article.

Authors' contributions

All authors contributed equally to the writing of this paper. All authors read and approved the final manuscript.

\section{Acknowledgements}

The authors are very grateful to the anonymous referees for their valuable suggestions. This paper was supported by the National Natural Science Foundation of China (No. 11201081), and supported by the Natural Science Foundation of Jiangxi Province (No. 20142BAB201001).

Received: 17 August 2015 Accepted: 8 January 2016 Published online: 20 January 2016

\section{References}

1. Wang, J, Niu, P: Optimal partial regularity for weak solutions of nonlinear sub-elliptic systems in Carnot groups. Nonlinear Anal. TMA 72, 4162-4187 (2010)

2. Wang, J, Liao, D: Optimal partial regularity for sub-elliptic systems with sub-quadratic growth in Carnot groups. Nonlinear Anal. TMA 75, 2499-2519 (2012)

3. De Giorgi, E: Un esempio di estremali discontinue per un problem variazionale di upo ellitico. Boll. Unione Mat. Ital. 4, 135-137 (1968)

4. Giaquinta, M: Multiple Integrals in the Calculus of Variations and Nonlinear Elliptic Systems. Princeton University Press, Princeton (1983)

5. Chen, Y, Wu, L: Second Order Elliptic Equations and Elliptic Systems. Science Press, Beijing (2003)

6. Duzaar, F, Steffen, K: Optimal interior and boundary regularity for almost minimizers to elliptic variational integrals. J. Reine Angew. Math. 546, 73-138 (2002)

7. Duzaar, F, Grotowski, JF: Partial regularity for nonlinear elliptic systems: the method of A-harmonic approximation. Manuscr. Math. 103, 267-298 (2000)

8. Duzaar, F, Grotowski, JF, Kronz, M: Regularity of almost minimizers of quasi-convex variational integrals with subquadratic growth. Ann. Mat. Pura Appl. 184, 421-448 (2005)

9. Duzaar, F, Mingione, G: The $p$-harmonic approximation and the regularity of $p$-harmonic maps. Calc. Var. Partial Differ. Equ. 20, 235-256 (2004)

10. Duzaar, F, Mingione, G: Regularity for degenerate elliptic problems via $p$-harmonic approximation. Ann. Inst. Henri Poincaré, Anal. Non Linéaire 21, 735-766 (2004)

11. Chen, $S, T a n, Z$ : The method of $A$-harmonic approximation and optimal interior partial regularity for nonlinear elliptic systems under the controllable growth condition. J. Math. Anal. Appl. 335, 20-42 (2007)

12. Duzaar, F, Gastel, A: Nonlinear elliptic systems with Dini continuous coefficients. Arch. Math. 78, 58-73 (2002)

13. Duzaar, F, Gastel, A, Mingione, G: Elliptic systems, singular sets and Dini continuity. Commun. Partial Differ. Equ. 29, 1215-1240 (2004)

14. Qiu, Y: Optimal partial regularity of second order nonlinear elliptic systems with Dini continuous coefficients for the superquadratic case. Nonlinear Anal. TMA 75, 3574-3590 (2012)

15. Domokos, A: On the regularity of subelliptic $p$-harmonic functions in Carnot groups. Nonlinear Anal. TMA 69, 1744-1756 (2008)

16. Domokos, A: Differentiability of solutions for the non-degenerate $p$-Laplacian in the Heisenberg group. J. Differ. Equ. 204, 439-470 (2004)

17. Domokos, A: On the regularity of $p$-harmonic functions in the Heisenberg group. Ph.D. thesis, University of Pittsburgh (2004)

18. Capogna, L: Regularity of quasi-linear equations in the Heisenberg group. Commun. Pure Appl. Math. 50, 867-889 (1997)

19. Capogna, L: Regularity for quasilinear equation and 1-quasiconformal maps in Carnot groups. Math. Ann. 313, 263-295 (1999)

20. Manfredi, J, Mingione, G: Regularity results for quasilinear elliptic equations in the Heisenberg group. Math. Ann. 339, 485-544 (2007)

21. Mingione, G, Zatorska-Goldstein, A, Zhong, X: Gradient regularity for elliptic equations in the Heisenberg group. Adv. Math. 222, 62-129 (2009)

22. Capogna, L, Garofalo, N: Regularity of minimizers of the calculus of variations in Carnot groups via hypoellipticity of systems of Hörmander type. J. Eur. Math. Soc. 5, 1-40 (2003)

23. Shores, E: Regularity theory for weak solutions of systems in Carnot groups. Ph. D. thesis, University of Arkansas (2005)

24. Föglein, A: Partial regularity results for sub-elliptic systems in the Heisenberg group. Calc. Var. Partial Differ. Equ. 32, 25-51 (2008)

25. Lu, G: The sharp Poincaré inequality for free vector fields: an endpoint result. Rev. Mat. Iberoam. 10, 453-466 (1994) 